\title{
Application of Event Management System in Quality Evaluation of Characteristic Database
}

\author{
Lu Jiaqi \\ Football School, Xi'an Physical Education University, Xi’an, Shaanxi, 710065, China \\ email: jackie-lu1988@foxmail.com
}

Keywords: Characteristic Database, Quality Evaluation, Event Management System

\begin{abstract}
At present, there is no unified evaluation scheme for the unique database project of the domestic digital library, the evaluation method is not mature, and the statistical analysis workload of the staff is large. Based on the principle of activity management system, an Internet-based feature database quality evaluation system is built to automatically review the achievements of personnel and summarize the evaluation level of feature database. The efficiency of feature database management and quality management can be improved. Through reducing the construction cost, the construction hall and construction direction are defined.
\end{abstract}

\section{Introduction}

Characteristic database is an important part of database resources [1]. It is a centralized reflection of the unique resources of the library. This is the core resource to attract library users and improve social impact. It not only embodies the personality of the library, but also is an important brand of the library information service competitiveness. In recent years, in order to integrate and collect local characteristic document resources and highlight the collected characteristic resources, most libraries have established their own characteristic databases. In addition, in the process of building and managing characteristic database, the standard and method of building database are also published. The project management team of CALIS (China Academic Library Information System) special characteristics database has formulated the quality management measures of CALIS 15th year plan national university special characteristics library.

\section{Quality Control Status of Characteristic Database}

The quality management of the characteristic database is a system engineer's ring which can measure many factors including the appropriateness, practicability, systematization, expansibility and reciprocity of the database. However, at present, there is not a feasible evaluation method in the acceptance evaluation of domestic digital library characteristic database project [2]. Caris features, data specifications, and comprehensive comments on the data volume of each task according to the expert comment specifications. JALIS (Jiangsu Academic Library \&amp; information system, Jiangsu Higher Education Document Guarantee System) and many regions have the characteristics of database quality evaluation after CALIS practice. In addition to the adjustment work, the evaluation of statistical and analytical experts is also needed. Before determining the overall score of each characteristic database, the staff need to weight the statistical information of each expert score of each evaluation project according to certain rules. Feature database evaluation is a complex project, which contains many factors. How to give each evaluation index a reasonable weight is a difficult problem. In the practice of feature database management, after continuous discussion and research, the principle of event management system is applied to the quality evaluation of feature database, which greatly reduces the daily load of staff and improves the efficiency of evaluation[3]. It provides a reliable method for the scientific and reasonable evaluation of characteristic database quality. 


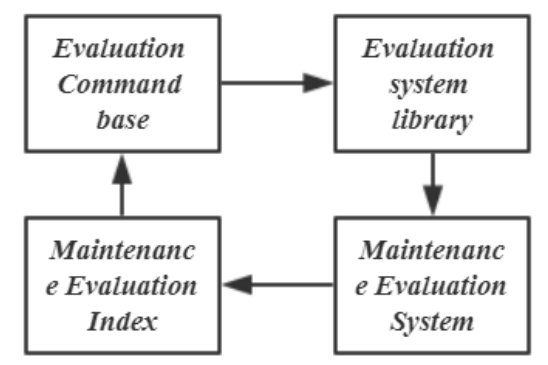

Figure 1 Quality assessment system

\section{The Setting of Event Management System in the Quality Evaluation of Characteristic Database}

Event management system is an information management system, which is designed to manage large-scale comprehensive events and complex sports events. In addition to basic information, personnel, project, venue, game arrangement, competition mechanism, constraint mechanism and other information management functions, it also has the function of competition arrangement and execution arrangement [6]. The characteristic database quality evaluation has many factors, such as data type, data quantity, metadata index, network environment and so on[4]. These factors influence and restrict each other. Using the principle of event management system to establish the quality evaluation system of characteristic database based on Internet can effectively improve the management level of characteristic database. This paper takes zadl (Zhejiang University electronic library, Zhejiang University Digital Library) characteristic database project as an example, introduces the application of event management system in the quality evaluation of characteristic database.

\subsection{Functional Module}

In the quality evaluation system of zadl characteristic database, the function modules are mainly composed of user center, foreground management, business management, event evaluation and system management. Management evaluation module, functional evaluation index, evaluation system, activity management, and related parameter setting of related evaluation module are completed[5]. The focus is on the parameter setting of the functional module, and the limitation of the permission authority of the experts who comment.

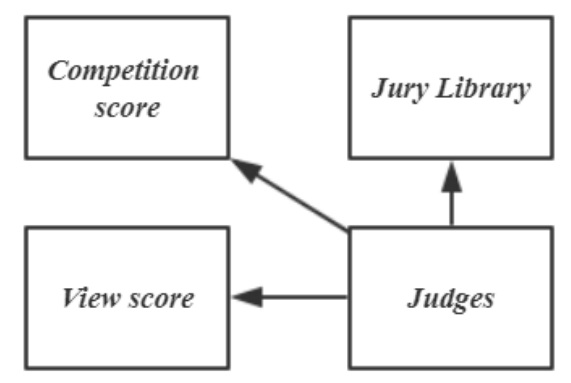

Figure 2 Scoring flow chart

In order to measure the construction quality of the self constructed characteristic database, we should investigate from all aspects and seek the opinions of experts, scholars and users in all fields. The setting of reference weight corresponding to the evaluation index can refer to the existing experience. The acceptance evaluation of zadl feature database mainly includes four items: database subject selection, resource content, quality, availability of feature database, and sustainable development of feature database. Each part contains many aspects. To set and edit the evaluation index, click the "evaluation index" button, input the evaluation index editing page (see Figure 2), input the corresponding information, and press the "send" button to finish. Among them, the "order" attribute is an effective input integer, so it is necessary that the order of system managers is the 
index management of the evaluation system. When the reviewers score, the index sequence page provides "percentage". In addition, it is necessary to enter a valid number (- 100) for all[6]. The judge's score result, and the final actual achievement, this value is repeated. Table 1 shows the benchmark weight setting corresponding to the evaluation index of zadl characteristic database.

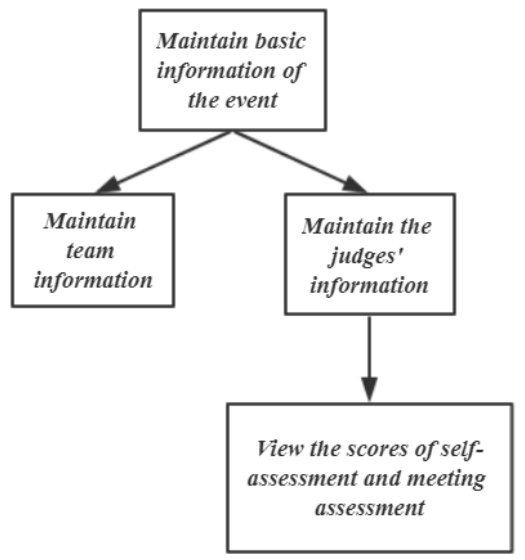

Figure 3 Workflow of quality assessment system

After adding evaluation indicators and corresponding reference weights on the evaluation indicator editing page, click the "evaluation system" button, and the system will automatically generate indicators and their hierarchies at all levels.

There are three steps to add an event: adding content, adding judges and adding teams. When adding an event, first select the evaluation system (which needs to be set in advance) and limit the judgment time of the event. Secondly, input the event content; thirdly, configure the expert information participating in the review and the feature database information to be reviewed.

Under this function, the administrator can add, modify and delete the information of the participating teams (that is, the characteristic database of participating in the evaluation), or click a project in the "participating team" to view the specific evaluation scores of each project.

\subsection{Workflow of Characteristic Database Quality Evaluation System}

According to the user name and password, the evaluation expert can log in to the characteristic database quality evaluation system and grade the participating databases[7]. Each evaluation index and corresponding reference component in the system are set by the manager in advance, so the final score of each participating data can be calculated automatically, and the final score can be determined based on the evaluation level of the basic feature database. Users of participating databases can log in to the evaluation system with effective user name and password, evaluate themselves according to the evaluation model, and improve the quality of database construction.

\section{The Application Effect of Event Management System in the Process of characteristic Database Quality Evaluation}

At the beginning of 2010, zadl characteristic resource management team began to develop characteristic database quality evaluation system based on event management system. After continuous discussion and research, the zadl local center (Zhejiang University) solicited opinions and suggestions from relevant experts and contractors of characteristic database. The system was completed in September 2010 and entered the trial operation stage[8]. In the trial operation stage, according to the feedback from all parties, the zadl feature resource management team constantly improves various functions of the system, striving to make the daily management and quality management of the feature database more scientific and standardized. On December 27, 2010, at the Zijingang Campus of Zhejiang University, Liu Xiaoqing (director of Zhejiang local education) opened the ribbon for zadl. As an important part of zadl, at the same time, the project of characteristic database is opened, and the quality evaluation system of characteristic database is 
formally used. According to the user name and password, each signature user can log in to the characteristic database quality evaluation system to display the latest notification and training information in real time. Or, using the self-evaluation function of the system, self-evaluation is carried out on the characteristic database established by ourselves to compare various evaluation parameters and reference weights. Commenting experts also said that user name and password login can evaluate parameters and system managers according to the set corresponding reference, according to each component of the comic project, the system will automatically obtain the final function of the database evaluation level, in order to give experts the results. In addition to ensuring the daily business of the system, the system manager also needs to maintain the evaluation index and benchmark weight according to the actual situation, understand the registration and selfevaluation of each host, the login and score of experts[9]. According to the unified deployment of zadl local center, zadl functional resource management team completed the first batch inspection of 15 feature database projects through feature database quality assessment system on April 9, 2011. Please complete the final inspection of the second batch of zadl for 18 characteristic database projects. In the evaluation process of the first badge of the feature database, the evaluation expert feature database quality evaluation system uses the participating feature database on the scenic spot, the collection system is the final result set by a set of programs. Which improves zadl. The efficiency of the characteristic resource management group is convenient for experts to review, and each contracting unit has a clear construction goal and work direction.

\section{Conclusion}

The daily management and quality characteristic quantity of activity management system database are introduced as evaluation, and the evaluation process of management activity process is investigated. It is found that the information of evaluation experts of system management personnel can not only be maintained, but also be maintained[10]. There are evaluation indexes and reference weights at any time. In addition, it has high flexibility and applicability because the registration and use methods of buildings can be known at any time. Database quality evaluation system is to automatically calculate the final results of each participating database. Its characteristics determine the quality level of database construction, improve management level, improve management efficiency, and save management cost.

\section{References}

[1] Shi, Congming, Deng, Hui, Liu, Yingbo,. High-performance Negative Database for Massive Data Management System of The Mingantu Spectral Radioheliograph. Publications of the Astronomical Society of the Pacific, vol. 129, no. 978, 2017.

[2] Li Zhang, Qianqian Zhang, Yaohua Tang,. LOVD ชै ASH: A Comprehensive LOVD Database Coupled with Diagnosis and an At - risk Assessment System for Hemoglobinopathies. Human Mutation, vol. 40, no. 12, 2019.

[3] Ma M, Diao K Y, Yang Z G, et al. Clinical associations of microvascular obstruction and intramyocardial hemorrhage on cardiovascular magnetic resonance in patients with acute ST segment elevation myocardial infarction (STEMI): An observational cohort study, vol. 97, no. 30, pp. e11617, 2018.

[4] Q. Liu, Y. Li, S. Hu,. Study on Filtering Mechanism and Operating Characteristic of the Controllably Inductive Power Filtering System. Transactions of China Electrotechnical Society, vol. 33, no. 14, pp. 3274-3283, 2018.

[5] Samrah Ahmed, Muireann Irish, Clare Loane,. Association between precuneus volume and autobiographical memory impairment in posterior cortical atrophy: Beyond the visual syndrome. Neuroimage Clinical, vol. 18, pp. 822-834, 2018. 
[6] Itayem, Deeyar A, Sladen, Douglas, Driscoll, Colin L,. Cochlear Implant Associated Labyrinthitis: A Previously Unrecognized Phenomenon With a Distinct Clinical and Electrophysiological Impedance Pattern. Otology \& Neurotology, vol. 38, 2017.

[7] Mazilu, Doina Carmen, Zazu, Mariana, Nedelcu, Viorica,. Effectiveness of pain management educational interventions on nurses' knowledge and attitudes regarding postoperative pain management:, a systematic review protocol. Jbi Database System Rev Implement Rep, vol. 16, no. 2, pp. 303-307, 2018.

[8] Carrasco R A, Blasco M F, García-Madariaga J, et al. Integrating a Tourism Service Quality Evaluation Linguistic Multi-criteria Decision Making Model into a Relational Database Management System, 2017.

[9] Viswam Subeesh, Hemendra Singh, Eswaran Maheswari,. Novel Adverse Events of Vortioxetine: A Disproportionality Analysis in USFDA Adverse Event Reporting System database. Asian Journal of Psychiatry, vol. 30, pp. 152, 2017.

[10] ZOU Tailong, ZHANG Xuemin,. Faculty of Education, A discussion on the antinomy of China's scientific research evaluation system: taking Tu Youyou's Nobel Prize winning as an example. Journal of Higher Education Management, 2017. 